\title{
Mass-to-Charge Ratio
}

National Cancer Institute

\section{Source}

National Cancer Institute. Mass-to-Charge Ratio. NCI Thesaurus. Code C48049.

The abbreviation $\mathrm{m} / \mathrm{z}$ is used to denote the dimensionless quantity formed by dividing the mass number of an ion by its charge number. It has long been called the mass-to-charge ratio although $\mathrm{m}$ is not the ionic mass nor is $\mathrm{z}$ a multiple or the elementary (electronic) charge e. (from IUPAC Compendium) 